\title{
Extra force and extra mass from noncompact Kaluza-Klein theory in a cosmological model
}

\author{
${ }^{1}$ José Edgar Madriz Aguilar* and ${ }^{2}$ Mauricio Bellini ${ }^{\dagger}$ \\ ${ }^{1}$ Instituto de Física y Matemáticas, AP: 2-82, (58040) Universidad Michoacana de San Nicolás \\ de Hidalgo, Morelia, Michoacán, México. \\ ${ }^{2}$ Departamento de Física, Facultad de Ciencias Exactas y Naturales, Universidad Nacional de \\ Mar del Plata and Consejo Nacional de Ciencia y Tecnología (CONICET), Funes 3350, (7600) \\ Mar del Plata, Argentina.
}

\begin{abstract}
Using the Hamilton-Jacobi formalism, we study extra force and extra mass in a recently introduced noncompact Kaluza-Klein cosmological model. We examine the inertial 4D mass $m_{0}$ of the inflaton field on a $4 \mathrm{D}$ FRW bulk in two examples. We find that $m_{0}$ has a geometrical origin and antigravitational effects on a non inertial $4 \mathrm{D}$ bulk should be a consequence of the motion of the fifth coordinate with respect to the $4 \mathrm{D}$ bulk.
\end{abstract}

Pacs numbers: 04.20.Jb, 11.10.kk, 98.80.Cq

\section{INTRODUCTION}

The extra dimension is already known to be of potential importance for cosmology $[1,2]$. During the last years there were many attempts to construct a consistent brane world (BW) cosmology [3]. On the other hand, the induced-matter, or space-time-matter (STM) theory stands out for its closeness to the Einstein's project of considering matter and radiation as manifestations of pure geometry [4]. Indeed, the gist of the whole theory is to assert that, by embedding the ordinary space-time into a five-dimensional vacuum space, it is possible to describe the macroscopic properties of matter in geometrical terms. Picking up several examples of cosmological and gravitational models, the theory shows how interpret the energy-momentum tensor corresponding to some standard matter configurations in terms of the geometry of the five-dimensional vacuum-space. It was recently questioned in a recent article [5]. The induced matter theory is sometimes given the name of Kaluza-Klein noncompact field gravity, since Klein's compactness condition [6] is dropped from the basic assumptions of the theory.

\footnotetext{
*E-mail address: edgar@itzel.ifm.umich.mx

${ }^{\dagger}$ E-mail address: mbellini@mdp.edu.ar
} 
The idea that matter in four dimensions (4D) can be explained from a 5D Ricci-flat $\left(R_{A B}=0\right)$ Riemannian manifold is a consequence of the Campbell's theorem. It says that any analytic $N$-dimensional Riemannian manifold can be locally embedded in a $(N+1)$ dimensional Ricci-flat manifold. This is of great importance for establishing the generality of the proposal that 4D field equations with sources can be locally embedded in 5D field equations without sources [7]. On the other hand, recently was remarked by Anderson that such that theorem lends only inadequate support, both because it offers no guarantee of continuous dependence on the data and because it disregards causality [5]. In his paper he point out that the theorem is only valid for analytic functions which renders it inappropiate for the study of the relativistic field equations in modern physics. However, analytic functions seems only inappropiate to describe some topological defects like black holes, but not to study cosmological models for which the manifold is global and there are no singularities. Other recent contribution to this issue was made by Katzourabis [8], who demonstrated a global generalization of the Campbell's theorem, removing the asumption of locality: Any pseudo-Riemannian analytic $N$-manifold can be embedded (the whole structure) naturally and isometrically into $(N+1)$-bulk with arbitrarily prefixed topological structure, fibred over the given, with vanishing Ricci curvature and torsion of some connection locally compatible with a global metric on the bulk. More generally he found that any $4 D$-manifold with a global tensor field representing the solution of $4 D$ General Relativity equations can be embedded naturally and isometrically into $(4+d)$-manifold $(d \in \mathbf{N})$ Ricci flat and torsion free, where the global topology, the differential structure and the dimensionality of the bulk can be fixed arbitrarily. In few words, 4D phenomenological matter can be induced by a $5 \mathrm{D}$ apparent vacuum in the framework of cosmological models where the universe is described by a 3D spatially isotropic, homogeneous and flat (analytic) metric.

An important question in STM and BW theories can be split up in two parts. 1) How to extract the correct 4D interpretation from geometrical objects, like scalar fields which appear in more than four dimensions. 2) How to predict observable effects from the extra dimensions. There are at least two ways to measure the effect, one which shows no change [9] and one which does [10]. For example, an attempt to understand the first of these questions in the framework of inflationary cosmology [which is governed by the neutral scalar (inflaton) field], from a 5D flat Riemannian manifold was made in [11].

In the last years extra force and extra mass has been subject of study [12]. It should be an observable effect from extra dimensions on the $4 \mathrm{D}$ spacetime. The aim of this work is to extend the Hamilton-Jacobi formalism developed by Ponce de Leon [9] to cosmological models where the expansion of the universe is governed by a single inflaton field. This interpretation has the advantage of being free of the complications and ambiguities of the geodesic approach. The formalism provides an unambiguous expression for the rest mass and its variation along the motion as observed in $4 \mathrm{D}$. It is independent of the coordinates and any parametrization used along the motion.

\section{COSMOLOGICAL APPROACH}

In a cosmological context, the energy of scalar fields has been argued to contribute to the expansion of the universe [13], and has been proposed to explain inflation [14] as well as the presently accelerated expansion [15]. In this paper we shall consider a cosmological model 
governed by a neutral scalar field that initially suffers an inflationary expansion that has a change of phase towards a decelerated (radiation and later matter dominated) expansion that thereinafter evolves towards the observed present day (quintessential) expansion.

We consider the recently introduced [16] 5D metric

$$
d S^{2}=\epsilon\left(\psi^{2} d N^{2}-\psi^{2} e^{2 N} d r^{2}-d \psi^{2}\right)
$$

where $d r^{2}=d x^{2}+d y^{2}+d z^{2}$. In order to describe cosmological models we shall consider a $3 \mathrm{D}$ spatially isotropic, homogeneous and flat metric (i.e., $x^{2}={ }^{2}=z^{2}$ ). Here, the coordinates $(N, \vec{r})$ are dimensionless, the fifth coordinate $\psi$ has spatial unities and $\epsilon$ is a dimensionless parameter that can take the values $\epsilon=1,-1$. The metric (1) describes a flat 5D manifold in apparent vacuum $\left(G_{A B}=0\right)$. With the aim to describe neutral matter in a $5 \mathrm{D}$ geometrical vacuum (1), as in the paper [17], we can consider the Lagrangian

$$
{ }^{(5)} \mathrm{L}(\varphi, \varphi, A)=-\sqrt{\left|\frac{{ }^{(5)} g}{{ }^{(5)} g_{0}}\right|}{ }^{(5)} \mathcal{L}(\varphi, \varphi, A),
$$

where $\left|{ }^{(5)} g\right|=\psi^{8} e^{6 N}$ is the absolute value of the determinant for the $5 \mathrm{D}$ metric tensor with components $g_{A B}(A, B$ take the values $0,1,2,3,4)$ and $\left.\right|^{(5)} g_{0} \mid=\psi_{0}^{8} e^{6 N_{0}}$ is a constant of dimensionalization determined by $\left.\right|^{(5)} g \mid$ evaluated at $\psi=\psi_{0}$ and $N=N_{0}$. We shall consider $N_{0}=0$, so that ${ }^{(5)} g_{0}=\psi_{0}^{8}$. We define the vacuum as a purely kinetic 5D-lagrangian on a globally 5D-flat metric [in our case, the metric (1)] [17]. Since the 5D metric (1) describes a manifold in apparent vacuum, the density Lagrangian $\mathcal{L}$ in (2) must to be

$$
{ }^{(5)} \mathcal{L}(\varphi, \varphi, A)=\frac{1}{2} g^{A B} \varphi, A \varphi, B,
$$

which represents a free scalar field. In the 3D comoving frame $U^{r}=0$, the geodesic dynamics $\frac{d U^{C}}{d S}=-\Gamma_{A B}^{C} U^{A} U^{B}$ with $g_{A B} U^{A} U^{B}=1$, give us the velocities

$$
U^{\psi}=-\frac{1}{\sqrt{u^{2}(N)-1}}, \quad U^{r}=0, \quad U^{N}=\frac{u(N)}{\psi \sqrt{u^{2}(N)-1}},
$$

which are satisfied for $S(N)= \pm|N|$. We shall consider the case $S(N)=|N|$. In this representation $\frac{d \psi}{d N}=\psi / u(N)$. Thus the fifth coordinate evolves as

$$
\psi(N)=\psi_{0} e^{\int d N / u(N)} .
$$

Here, $\psi_{0}$ is a constant of integration that has spatial unities. From the mathematical point of view, we are taking a foliation of the 5D metric (1) with $r$ constant.

In order to obtain equations of practical use, we can introduce the action $\mathcal{S}\left(x^{A}\right)$ as a function of the generalyzed coordinates $x^{A}$. Hence, since the momentum $P^{A}=-\frac{\partial \mathcal{S}}{\partial x^{A}}$, for a diagonal tensor metric $g^{A B}$ we obtain the Hamilton-Jacobi equation

$$
g^{A B}\left(\frac{\partial \mathcal{S}}{\partial x^{A}}\right)\left(\frac{\partial \mathcal{S}}{\partial x^{B}}\right)=M_{(5)}^{2},
$$


where $M_{(5)}$ is the invariant 5D gravitational mass of the object under study (in our case, the mass of the inflaton field). In the particular frame (4), with the Lagrangian (2) and (3), $M_{(5)}$ describes the 5D mass of the scalar field $\varphi$ in the frame (4). In this case the tensor metric is symmetric (and diagonal), and the Hamilton-Jacobi equation (6) adopts the particular form

$$
g^{N N}\left(\frac{\partial \mathcal{S}}{\partial \varphi, N}\right)^{2}+g^{\psi \psi}\left(\frac{\partial \mathcal{S}}{\partial \varphi_{, \psi}}\right)^{2}=M_{(5)}^{2} .
$$

\section{EXTRA FORCE AND EXTRA MASS}

In general, the line element (1) can be written as:

$$
d S^{2}=d s^{2}+d S_{(4)}^{2},
$$

where $d s^{2}$ describes the $4 \mathrm{D}$ line element and $d S_{(4)}^{2}$ only the line element related with the fifth coordinate. We shall define the extra force

$$
F^{e x t}=\frac{d P^{x^{4}}}{d s}
$$

as the force on the sub manifold $d s^{2}$ due to the motion of the fifth coordinate. In general, $P^{x^{4}}$ is defined as

$$
P^{x^{4}}=\frac{\partial^{(5)} L}{\partial \varphi_{, x^{4}}}
$$

In the frame (4) $P^{x^{4}} \equiv P^{\psi}$, and is given by $P^{\psi}=-\frac{\psi^{4} e^{3 N}}{\psi_{0}^{2}}\left(g^{\psi \psi}\right)^{2} \varphi_{, \psi}$, which also can be written in terms of the potential

$$
P^{\psi}=-\frac{\psi^{4} e^{3 N}}{\psi_{0}^{2}} g^{\psi \psi} \frac{\partial V(\varphi)}{\partial \varphi_{, \psi}},
$$

where $V(\varphi)$ depends on the particular frame of the observer. In the next subsections we shall see different forms of it in two different frames. Hence, in the frame (4) the extra force holds

$$
F^{e x t}=\frac{\psi^{3} e^{3 N}}{\psi_{0}^{2}}\left(3 \frac{\star}{\psi} \varphi_{, \psi}+3 \varphi_{, \psi}+\stackrel{\star}{\varphi}_{, \psi}\right)
$$

where the overstar denotes the derivative with respect to $N$.

On the other hand, from the equation $g_{A B} U^{A} U^{B}=1$, we obtain the invariant 5D mass $M_{(5)}$

$$
g_{A B} P^{A} P^{B}=M_{(5)}^{2}
$$

where $P^{A}=M_{(5)} U^{A}$ is the momentum. For example, in the frame (4) the 4D mass $m_{0}$ and the 5D invariant mass $M_{(5)}$ are given respectively by 


$$
M_{(5)}^{2}=g^{N N}\left(\frac{\partial \mathcal{S}}{\partial \varphi_{, N}}\right)^{2}+g^{\psi \psi}\left(\frac{\partial \mathcal{S}}{\partial \varphi_{, \psi}}\right)^{2}, \quad m_{0}^{2}=g^{N N}\left(\frac{\partial \mathcal{S}}{\partial \varphi_{, N}}\right)^{2}
$$

so that its diference

$$
m_{0}^{2}-M_{(5)}^{2}=-g^{\psi \psi}\left(\frac{\partial \mathcal{S}}{\partial \varphi, \psi}\right)^{2}
$$

is nonzero. The interesting here is that $m_{0}^{2}>M_{(5)}^{2}$. In other words, in the frame (4) the motion of the fifth coordinate has an antigravitational effect on the field $\varphi$ on the submanifold (or bulk) $d s^{2}$. In the next sections we shall study some examples which could be relevant in cosmological models.

\section{A. A 4D FRW cosmology}

We consider the transformations: $t=\int \psi(N) d N, R=r \psi, L=\psi(N) e^{-\int d N / u(N)}$, such that for $\psi(t)=1 / h(t)$, we obtain the 5D metric $d S^{2}=\epsilon\left(d t^{2}-e^{2 \int h(t) d t} d R^{2}-d L^{2}\right)$, where $L=\psi_{0}$ is a constant and $h^{2}(t)=(\dot{b} / b)^{2}=(8 / 3) \pi G\langle\rho\rangle$ is the effective Hubble parameter defined from the effective scale factor of the universe $b$. In this frame $(R, t, L)$, the velocities $\hat{U}^{A}=\frac{\partial \hat{x}^{A}}{\partial x^{B}} U^{B}$, are

$$
U^{t}=\frac{2 u(t)}{\sqrt{u^{2}(t)-1}}, \quad U^{R}=-\frac{2 r}{\sqrt{u^{2}(t)-1}}, \quad U^{L}=0,
$$

where the old velocities $U^{B}$ are $U^{N}, U^{r}=0$ and $U^{\psi}$ and the velocities $\hat{U}^{B}$ are constrained by the condition

$$
\hat{g}_{A B} \hat{U}^{A} \hat{U}^{B}=1 .
$$

The variables $(t, R, L)$ has physical meaning, because $t$ is the cosmic time and $(R, L)$ are spatial variables. Since the line element is a function of time $t$ (i.e., $S \equiv S(t)$ ), the new coordinate $R$ give us the physical distance between galaxies separated by cosmological distances: $R(t)=r / h(t)$. Note that for $r>1(r<1)$, the 3D spatial distance $R(t)$ is defined on super (sub) Hubble scales. Furthermore $b(t)$ is the effective scale factor of the universe and describes its effective 3D Eucliden (spatial) volume. Hence, the effective 4D metric is a spatially (3D) flat FRW one

$$
d S^{2} \rightarrow d s^{2}=\epsilon\left(d t^{2}-e^{2 \int h(t) d t} d R^{2}\right)
$$

and has a effective $4 \mathrm{D}$ scalar curvature ${ }^{(4)} \mathcal{R}=6\left(\dot{h}+2 h^{2}\right)$. The metric $(18)$ has a metric tensor with components $g_{\mu \nu}(\mu, \nu$ take the values $0,1,2,3)$. The absolute value of the determinant for this tensor is $\left|{ }^{(4)} g\right|=\left(b / b_{0}\right)^{6}$. The density Lagrangian in this new frame was obtained in a previous work [17]

$$
{ }^{(4)} \mathcal{L}[\varphi(\vec{R}, t), \varphi, \mu(\vec{R}, t)]=\frac{1}{2} g^{\mu \nu} \varphi_{, \mu} \varphi_{, \nu}-\frac{1}{2}\left[(R h)^{2}-\frac{b_{0}^{2}}{b^{2}}\right]\left(\nabla_{R} \varphi\right)^{2},
$$


and the equation of motion for $\varphi$ yields

$$
\ddot{\varphi}+3 h \dot{\varphi}-\frac{b_{0}^{2}}{b^{2}} \nabla_{R}^{2} \varphi+\left[\left(4 \frac{h^{3}}{\dot{h}}-3 \frac{\dot{h}}{h}-3 \frac{h^{5}}{\dot{h}^{2}}\right) \dot{\varphi}+\left(\frac{b_{0}^{2}}{b^{2}}-h^{2} R^{2}\right) \nabla_{R}^{2} \varphi\right]=0 .
$$

From eqs. (19) and (20), we obtain respectively the effective scalar 4 D potential $V(\varphi)$ and its derivative with respect to $\varphi(\vec{R}, t)$

$$
\begin{aligned}
V(\varphi) & \equiv \frac{1}{2}\left[(R h)^{2}-\left(\frac{b_{0}}{b}\right)^{2}\right]\left(\nabla_{R} \varphi\right)^{2}, \\
V^{\prime}(\varphi) & \equiv\left(4 \frac{h^{3}}{\dot{h}}-3 \frac{\dot{h}}{h}-3 \frac{h^{5}}{\dot{h}^{2}}\right) \dot{\varphi}+\left(\frac{b_{0}^{2}}{b^{2}}-h^{2} R^{2}\right) \nabla_{R}^{2} \varphi,
\end{aligned}
$$

where the prime denotes the derivative with respecto to $\varphi$. The equations (19) and (20) describe the dynamics of the inflaton field $\varphi(\vec{R}, t)$ in a metric $(18)$ with a Lagrangian

$$
{ }^{(4)} \mathcal{L}[\varphi(\vec{R}, t), \varphi, A(\vec{R}, t)]=-\sqrt{\left|\frac{{ }^{(4)} g}{{ }^{(4)} g_{0}}\right|}\left[\frac{1}{2} g^{\mu \nu} \varphi_{, \mu} \varphi_{, \nu}+V(\varphi)\right],
$$

where $\left|{ }^{(4)} g_{0}\right|=1$.

Furthermore, the function $u$ can be written as a function of time $u(t)=-\frac{h^{2}}{\grave{h}}$, where the overdot represents the derivative with respect to the time. The solution $N=\operatorname{arctanh}[1 / u(t)]$ corresponds to a time dependent power-law expanding universe $h(t)=p(t) t^{-1}$, such that the effective scale factor go as $b \sim e^{\int p(t) / t d t}$. A model for the evolution of the universe was recently developed in [18]. Furthermore, the 4D energy density $\rho$ and the pressure p are [16]

$$
\begin{aligned}
& 8 \pi G \rho=3 h^{2}, \\
& 8 \pi G \mathrm{p}=-\left(3 h^{2}+2 \dot{h}\right) .
\end{aligned}
$$

Note that the function $u(t)$ can be related to the deceleration parameter $q(t)=-\ddot{b} b / \dot{b}^{2}$ : $u(t)=1 /[1+q(t)]$. In what follows we shall consider $\epsilon=1$ when the universe is accelerated $(q<0)$ and $\epsilon=-1$ in epochs when it is decelerated $(q>0)$. From the condition (17) we can differentiate some different stages of the universe. If $u^{2}(t)=\frac{4 r^{2}\left(b / b_{0}\right)^{2}-1}{3}>1$, we obtain that $r$ can take the values $r>1(r<1)$ for $b / b_{0}<1\left(b / b_{0}>1\right)$, respectively. Note that now the condition (17) implies that $r \equiv r(t)$, for a given $h(t)$. In this case $q<0$, so that the expansion is accelerated. On the other hand if $u^{2}(t)=\frac{4 r^{2}\left(b / b_{0}\right)^{2}-1}{3}<1, r$ can take the values $r<1(r>1)$ for $b / b_{0}>1\left(b / b_{0}<1\right)$, respectively. In this stage $q>0$ and the expansion of the universe is decelerated, so that the function $u(t)$ take the values $0<u(t)<1$ and the velocities $(16)$ become imaginary. Thus, the metric $(18)$ shifts its signature from $(+,-,-,-)$ to $(-,+,+,+)$. When $u(t)=1$ the deceleration parameter becomes zero because $\ddot{b}=0$. At this moment the velocities (16) rotates sincronically in the complex plane and $r$ take values $r=1$ or $r<1$ for $b / b_{0}=1$ or $b / b_{0}>1$, respectively. On the other hand, the effective 4D energy density operator $\rho$ is

$$
\rho=\frac{1}{2}\left[\dot{\varphi}^{2}+\frac{b_{0}^{2}}{b^{2}}(\nabla \varphi)^{2}+2 V(\varphi)\right] .
$$


Hence, the 4D expectation value of the Einstein equation $\left(\frac{\dot{b}}{b}\right)^{2}=\frac{8 \pi G}{3} \rho$ on the 4D FRW metric (18), will be

$$
\left\langle h^{2}\right\rangle=\frac{4 \pi G}{3}\left\langle\dot{\varphi}^{2}+\frac{b_{0}^{2}}{b^{2}}(\nabla \varphi)^{2}+2 V(\varphi)\right\rangle,
$$

where $G$ is the gravitational constant and $\left\langle H^{2}\right\rangle \equiv h^{2}=\dot{b}^{2} / b^{2}$.

In this frame the 5D momentum $P^{L}$ is null: $P^{L}=0$. This implies that the extra force will be

$$
F_{\text {ext }}=0
$$

It also can be viewed from the point of view of the extra mass. In this frame $m_{0}^{2}=M_{(5)}^{2}$ where

$$
\left(\frac{\partial \mathcal{S}}{\partial \varphi_{, t}}\right)^{2}-e^{2 \int h(t) d t}\left(\frac{\partial \mathcal{S}}{\partial \varphi_{, R}}\right)^{2}=M_{(5)}^{2} .
$$

Hence, the inertial $4 \mathrm{D}$ mass $m_{0}$ is the same than the invariant $5 \mathrm{D}$ mass $M_{(5)}$, so that there is not extra force on the effective $4 \mathrm{D}$ frame. This can be justified from the fact that the fifth coordinate $L$ do not varies in this frame. In other words the $4 \mathrm{D}$ bulk $d s^{2}$ is the same that the 5D manifold $d S^{2}$, because $d S_{(4)}=0$ for an observer that "expands with the universe" in an inertial frame.

\section{B. A frame with variable fifth coordinate}

Other interesting frame can be described by means of the transformation $t=\int \psi(N) d N$, $R=r \psi$ and $\xi=\psi(N) e^{\int H^{\star}(N) / H(N) d N}$, so that the $5 \mathrm{D}$ velocities are

$$
\begin{aligned}
U^{t} & =\frac{2 u(t)}{\sqrt{u^{2}(t)-1}}, \\
U^{R} & =-\frac{2 r}{\sqrt{u^{2}(t)-1}}, \\
U^{\xi} & =\frac{u(t)}{\sqrt{u^{2}(t)-1}}\left(\frac{\dot{H}}{h H}-\frac{\dot{h}}{h^{2}}\right) \frac{H}{H_{0}} .
\end{aligned}
$$

In this frame the 5D line element is given by

$$
d S^{2}=\epsilon\left(d t^{2}-e^{2 \int h(t) d t} d R^{2}-\left(\frac{H_{0}}{H}\right)^{2} d \xi^{2}\right),
$$

where the $4 \mathrm{D}$ line element (or "bulk") $d s^{2}$ is given by the first two terms in (33)

$$
d s^{2}=\epsilon\left(d t^{2}-e^{2 \int h(t) d t} d R^{2}\right)
$$


and $h^{2}(t)=H^{2}(t)+\frac{C}{3}$ for a given constant $C$. Hence, the extra force on the $4 \mathrm{D}$ bulk will be $F^{e x t}=\frac{d P^{\xi}}{d s}$. Note that extra force becomes from the motion of the fifth coordinate in the effective $4 \mathrm{D}$ bulk. In other words, an observer in the $4 \mathrm{D}$ bulk (34), will move under the influence of an extra force that, in the example here studied, takes the form

$$
F^{e x t}=\left(\left|1-\frac{r^{2} \dot{h}^{2}}{h^{4}} e^{2 \int h d t}\right|\right)^{-1 / 2} \frac{d P^{\xi}}{d t}
$$

which is invariant under changes of signature (i.e., $\epsilon=1 \rightarrow \epsilon=-1$ ). The 5D Lagrangian in this frame takes the form

$$
{ }^{(5)} L(\varphi, \varphi, A)=-\left(\frac{b}{b_{0}}\right)^{3} \frac{H_{0}}{H}\left(\frac{1}{2} g^{\alpha \beta} \varphi_{, \alpha} \varphi_{, \beta}+V(\varphi)\right),
$$

so that the momentum $P^{\xi}$ is

$$
P^{\xi}=-\left(\frac{b}{b_{0}}\right)^{3} \frac{H_{0}}{H} g^{\xi \xi} \frac{\partial V(\varphi)}{\partial \varphi_{, \xi}}
$$

In this representation the potential $V(\varphi)$ assumes the form

$$
V(\varphi)=\frac{1}{2}\left[(R h)^{2}-\left(\frac{b_{0}}{b}\right)^{2}\right]\left(\nabla_{R} \varphi\right)^{2}-\frac{1}{2}\left(\frac{H}{H_{0}}\right)^{2} \varphi_{, \xi}^{2}-\frac{H}{H_{0}}(R h) \varphi_{, \xi} \nabla_{R} \varphi
$$

so that the momentum $P^{\xi}$ is

$$
P^{\xi}=\left(\frac{b}{b_{0}}\right)^{3}\left[\left(\frac{H}{H_{0}}\right) \varphi_{, \xi}+(R h) \nabla_{R} \varphi\right] .
$$

Note that the effective kinetic component in the 5D Lagrangian (36) is 4D, but the potential (38) is evaluated in 5D frame (30),(31),(32). From eqs. (35) and (39), we obtain the extra force for this frame

$$
\begin{aligned}
F^{e x t} & =\left(\frac{b}{b_{0}}\right)^{3}\left[\left|1-\left(\frac{R \dot{h}}{h}\right)^{2}\left(\frac{b}{b_{0}}\right)^{2}\right|\right]^{-1 / 2}\left[\left(3 \frac{\dot{b}}{b} \frac{H}{H_{0}}+\frac{\dot{H}}{H}\right) \varphi_{, \xi}+\left(3 \frac{\dot{b}}{b}(R h)+(\dot{R} h+R \dot{h})\right) \nabla_{R} \varphi\right. \\
& \left.+\frac{H}{H_{0}} \frac{d}{d t}\left(\varphi_{, \xi}\right)+(R h) \frac{d}{d t}\left(\nabla_{r} \varphi\right)\right],
\end{aligned}
$$

where $\left(\frac{b}{b_{0}}\right)^{2}=e^{2 \int h d t}$. The important fact here is that the extra force is originated in the last two terms of the 5D potential (38), which depends on the fifth coordinate $\xi$.

On the other hand the $4 \mathrm{D}$ squared mass of the inflation field $\varphi$ on the $4 \mathrm{D}$ bulk (34), is given by

$$
m_{0}^{2}=\left(\frac{\partial \mathcal{S}}{\partial \varphi_{, t}}\right)^{2}-e^{-2 \int h d t}\left(\frac{\partial \mathcal{S}}{\partial \varphi_{, R}}\right)^{2}
$$

so that one obtains 


$$
m_{0}^{2}-M_{(5)}^{2}=\left(\frac{H}{H_{0}}\right)^{2}\left(\frac{\partial \mathcal{S}}{\partial \varphi_{, \xi}}\right)^{2}
$$

which gives $m_{0}^{2} \geq M_{(5)}^{2}$ because the right hand of the equation (42) is positive (for $C>0$ ). This is an important result which shows that the motion of the fifth coordinate has an antigravitational effect on a observer in a $4 \mathrm{D}$ bulk in which the inflaton field has a $4 \mathrm{D}$ mass $m_{0}$. This fact should be responsible for the extra force (40) because the observer "is placed" in a non inertial frame (or 4D bulk). In this framework the motion of the fifth coordinate is viewed on the bulk as an extra force. Note that it becomes zero as $C \rightarrow 0$, because in this limit $U^{\xi} \rightarrow 0$ and $V(\varphi) \rightarrow \frac{1}{2}\left[(R h)^{2}-\left(\frac{b_{0}}{b}\right)^{2}\right]\left(\nabla_{R} \varphi\right)^{2}$. On the other hand, $U^{\xi} \rightarrow 0$ as $t \rightarrow \infty$, because $\dot{H}<0$ (and $\dot{h}<0$ ) along all the history of the universe, such that $\left(\frac{H}{H_{0}}\right)_{t \rightarrow \infty} \rightarrow 0$. Hence, for very late times the external force (40) on the bulk becomes negligible. However, this force should be very important in the early universe when $H / H_{0} \gg 1$ and the equation of state is $\mathrm{p} \simeq-\rho$, being $\mathrm{p}$ and $\rho$ respectively the pressure and energy density. Note that $H_{0}$ is the value of the Hubble parameter at the end of inflation.

To ilustrate the formalism we can consider the case where $h(t)=t^{-1} p_{1}(t)$ and $H(t)=$ $t^{-1} p(t)$, where

$$
\begin{aligned}
& p_{1}(t)=\sqrt{\left(2 / 3+A t^{-2}-B t^{-1}\right)^{2}+\frac{C}{3} t^{2}}, \\
& p(t)=\sqrt{2 / 3+A t^{-2}-B t^{-1}}
\end{aligned}
$$

Here $A=1.510^{30} \mathrm{G}^{1}, B=10^{15} \mathrm{G}^{1 / 2}$ and we take the special case where the constant $C$ is the cosmological constant $\Lambda: \Lambda=1.510^{-121} \mathrm{G}^{-1}$. Furthermore, $G=M_{p}^{-2}$ is the gravitational constant and $M_{p}=1.2 \times 10^{19} \mathrm{GeV}$ is the Planckian mass [18]. The function $p_{1}(t)$ represents the power of expansion of the universe in Planckian times. For early times $p_{1}(t) \gg 1$ and the universe is accelerated $(\ddot{b}>0)$. At $t_{r} \simeq 10^{16} \mathrm{G}^{1 / 2}$, after inflation ends, the universe becomes radiation dominated: $p_{1}\left(t_{r}\right) \simeq 1 / 2$ and after it (for $t \gg t_{r}$ ) matter dominated: $p_{1}\left(t \gg t_{r}\right) \simeq 2 / 3$. However, at $t \simeq 10^{60.22} \mathrm{G}^{1 / 2}$ the cosmological constant begins to be determinant and the universe becomes almost vacuum dominated (quintessential expansion): $\mathrm{p} \lesssim-\frac{2}{3} \rho$. The function $p(t)$ represents the power of expansion of the universe without the cosmological constant. The difference between $p_{1}$ and $p$ becomes notorious for very large time (i.e., for $t>10^{60.22} \mathrm{G}^{1 / 2}$ ). Numerical calculations give us the time for which $\ddot{b}=q=0$ at the end of inflation: $x\left(t_{0}\right) \simeq 14.778$ [we take $\left.x(t)=\log _{10}(t)\right]$. At this moment $N\left(t_{0}\right)=0$, but after it becomes positive. Furthermore, for $x(t)>x\left(t_{*}\right)$ [with $x\left(t_{*}\right) \simeq 60.22$ ], $p_{1}$ begins to increase from the value $p_{1} \simeq 2 / 3$ and the $4 \mathrm{D}$ bulk universe is accelerated. In other words, as we demonstrated in a prvious work [18], the present day observed (quintessential) acceleration of the universe is consequence of the nonzero $\Lambda$, and has been notorious since the universe was nearly 4 billion years old (when the supernovae explosions ocurred).

\section{FINAL COMMENTS}

In this work we have studied the possible origin of extra force and extra mass from a noncompact Kaluza-Klein formalism recently introduced by using the Hamilton-Jacobi formalism in the framework of cosmological models. However, it is one of the two possible 
outcomes to study this topic (see, for example [10]). We have examined the inertial 4D mass $m_{0}$ of the inflaton field on a $4 \mathrm{D}$ FRW bulk in two examples. In the first one $d s^{2}=d S^{2}$, so that the inertial mass $m_{0}$ is the same than the 5D gravitational mass $M_{(5)}$ of the inflaton field. As consequence of this fact there are no extra force on the $4 \mathrm{D}$ bulk. However, in the second example antigravitational effects on a non inertial 4D bulk should be a consequence of the motion of the fifth coordinate with respect to this bulk, because $d S^{2} \neq d s^{2}$ so that $m_{0}^{2}>M_{(5)}^{2}$. This disagreement between the 4D inertial and 5D gravitational masses is viewed on the $4 \mathrm{D}$ bulk as an extra force. The important here is that $m_{0}$ has a geometrical origin and depends on the frame of the observer. However, $M_{(5)}$ is a $5 \mathrm{D}$ invariant gravitational mass and do not depends on the frame of the observer. In other words, all test particles travel on five-dimensional geodesics but observers, who are bounded to spacetime, have access only to the 4D part of the trajectory. Finally, in the cosmological model here studied, we find that both, the discrepance between $m_{0}$ and $M_{(5)}$ and extra force (on cosmological scales), are bigger in the early universe [i.e., during inflation $(x(t)<14.778)$ ], but becomes negligible for large times. However, from the point of view of experimentation, at present

such that discrepance should be more notorious on astrophysical scales, where gravitational instabilities are important.

\section{Acknowledgements}

The authors acknowledge Dr. J. Ponce de Leon for useful ideas. JEMA acknowledges CONACyT and IFM of UMSNH (México) for financial support. MB acknowledges CONICET, AGENCIA and UNMdP (Argentina) for financial support. 


\section{REFERENCES}

[1] J. Ponce de León, Gen. Relativ. Gravit. 20539 (1998).

[2] J. M. Overduin and P. S. Wesson, Phys. Rep. 283, 303 (1997); M. J. Duff, Int. J. Mod. Phys. A11, 5623 (1996).

[3] N. Arkani-Hamed, S. Dimopoulos and G. Dvali, Phys. Lett.B429, 263 (1998); I. Antoniadis, N. A. Arkani-Hamed, S. Dimopoylos and G. Dvali, Phys. Lett. B436, 257 (1998); L. Randall and R. Sundrum, Phys. Rev. Lett. 83, 3370 (1999); L. Randall and R. Sundrum, Phys. Rev. Lett. 83, 4690 (1999); F. Dahia, C. Romero, Class. Quant. Grav. 21, 927 (2004).

[4] A. Einstein, The meaning of relativity, Princeton University Press, Princeton, 1956; J. Weeler, Einstein's vision, Springer, Berlin, 1968; A. Salam, Rev. Mod. Phys. 52, 525 (1968).

[5] E. Anderson, The Campbell-Magaard theorem is inadequate and inappropiate as a protective theorem for relativistic equation. E-print: gr-qc/0409122.

[6] T. Kaluza, Sitz. Preuss. Akad. Wiss, 33, 966 (1921); O. Klein, Z. Phys. 37, 895 (1926).

[7] P. S. Wesson, Space-Time-Matter, World Scientific, Singapore, 1999.

[8] N. I. Katzourakis, Bundly-Theoretical globalization of Campbell-Magaard embedding theorem in the context of MD gravity. E-print: math-ph/0407067.

[9] J. Ponce de Leon, Int. J. Mod. Phys. D12, 757 (2003); Gn. Rel. Grav. 35, 1365 (2003); Gen. Rel. Grav. 36, 1333 (2004). See also J. Ponce de Leon, Grav. Cosmol. 8, 272 (202).

[10] B. Mashhoon, P. S. Wesson and H. Liu, Gen. Rel. Grav. 30, 555 (1998); P. S. Wesson, B. Mashhoon, H. Liu, W. N. Sajko, Phys. Lett. 456, 34 (1999).

[11] Mauricio Bellini, Nucl. Phys. B660, 389 (2003).

[12] S. S. Seahra and P. S. Wesson, Gen. Rel. Grav. 33, 1731 (2001); F. Dahia, E. M. Monte, C. Romero, Mod. Phys. Lett. A18, 1773 (2003).

[13] J. Dreitlein, Phys. Rev. Lett. 33, 1243 (1974); H. W. van Holten, Phys. Rev. Lett. 89, 202301 (2002).

[14] A. Guth, Phys. Rev. D23, 347 (1981).

[15] R. R. Caldwell, R. Dave and P. J. Steinhardt, Phys. Rev. Lett. 80, 1582 (1998); C. Armendariz-Picon, V. Mukhanov and P. J. Steinhardt, Phys. Rev. Lett. 85, 4438 (2000).

[16] D. S. Ledesma and M. Bellini, Phys. Lett. B581, 1 (2004).

[17] J. E. Madriz Aguilar and M. Bellini, Eur. Phys. J. C38, 123 (2004); J. E. Madriz Aguilar and M. Bellini, Phys. Lett. B596, 116 (2004).

[18] J. E. Madriz Aguilar and M. Bellini, Eur. Phys. J. C38, 367 (2004). 